Baltic Astronomy, vol. 5, 379-390, 1996.

\title{
THE WBVR PHOTOMETRY OF BRIGHT NORTHERN STARS
}

V. Kornilov, A. Mironov and A. Zakharov

Sternberg Astronomical Institute, Moscow University, Universitetskij Prospect 13, Moscow 119899, Russia

Received August 15, 1995.

\begin{abstract}
Photoelectric measurements of more than 13500 stars have been made with a four-channel photometer attached to the 48 $\mathrm{cm}$ reflector of the Tien Shan High Altitude Station of the Sternberg Astronomical Institute during 1985-1988. The program, observational details, photometric equipment and reduction procedures used are described. Particular attention is paid to definition of the photometric WBVR system of the catalogue. The stability of the photometric system has been assured by taking into account temporal and temperature shifts of the photometric passbands. These shifts were obtained from the analysis of observational data. Estimates of the accuracy of the photometry are presented. A study of completeness of the catalogue shows that it contains more than $95 \%$ of stars with $V \leq 7$ mag. The transformation to the photometric system $U B V$ is discussed.
\end{abstract}

Key words: catalogs - methods: observational - techniques: photometric: $W B V R$ photometric system

\section{INTRODUCTION}

Photometric observations in the $W B V R$ system were started at the Sternberg Astronomical Institute in 1976. The choice of this broad-band photometric system was influenced by Straižys (1973) who has suggested it as an alternative to Johnson's $U B V$ system to avoid its shortcomings.

From 1976 till 1984, WBVR observations were obtained for more than 2000 stars with magnitudes in the range from 0 to $8 \mathrm{mag}$ 
(Mironov et al. 1984, Khaliullin et al. 1985). In 1984, we started a program of magnitude limited WBVR photometry of bright stars in the northern sky. The total number of the program stars was limited by the equipment efficiency, number of observers and the observation period planned (four years): it contained about 15 thousand stars down to $7.2 \mathrm{mag}$ in the $V$ passband.

During the period of observations, WBVR photometry of 13560 objects (stars and multiple systems) has been obtained in the range of declination from $-14^{\circ}$ to $+90^{\circ}$. The results have been published in the Catalogue of $W B V R$ Magnitudes of Bright Northern Stars by Kornilov et al. (1991).

\section{THE PROGRAM AND OBSERVATIONS}

The observations have been made at the Tien Shan High Altitude Station of the Sternberg Astronomical Institute near Alma-Ata, Kazakhstan (at the altitude of $3000 \mathrm{~m}$ above sea level) using a 48 $\mathrm{cm}$ reflector. The latitude of site is about $+43^{\circ}$. The site has a high transparency of the atmosphere (the contribution of aerosols in absorption is about $0.01 \mathrm{mag}$ on some nights) and good stability. The observations were started in January 1985 and completed in October 1988. The photometric measurements of all program stars were carried out during 360 nights with total observational time of 2400 hours. About 57000 independent measurements of program stars and more than 13000 measurements of standard stars have been obtained. The distribution of the observation time over years and seasons is listed in Table 1.

For observations, only the nights with complete absence of cloudiness and with a transparency variation less than $0.01 \mathrm{mag}$ during 20 minutes were used. Further analysis of the obtained data confirmed a sufficient stability of transparency for $95 \%$ of observational time. Only $0.5 \%$ of observational data were rejected due to instability of the atmospheric extinction.

About $65 \%$ of the observations were made in the autumn and winter seasons. The great majority of observations $(80 \%)$ was obtained at temperatures from $+5^{\circ} \mathrm{C}$ to $-15^{\circ} \mathrm{C}$ (i.e. at $-3.8^{\circ} \mathrm{C}$ on the average). Small temperature variations resulted in the homogeneity of the observational data. 
Table 1. Some characteristics of the observational conditions.

\begin{tabular}{lcccc}
\hline $\begin{array}{l}\text { Year or } \\
\text { season }\end{array}$ & $\begin{array}{c}\text { Number } \\
\text { of nights }\end{array}$ & $\begin{array}{c}\text { Full } \\
\text { time, h }\end{array}$ & $\begin{array}{c}\text { Number of } \\
\text { measurements }\end{array}$ & $\begin{array}{c}\text { Average } \\
\text { temperature } \\
{ }^{\circ} \mathrm{C}\end{array}$ \\
\hline 1985 & 85 & 570 & 10000 & -4.5 \\
1986 & 97 & 710 & 20000 & -4.0 \\
1987 & 88 & 540 & 17000 & -2.6 \\
1988 & 80 & 510 & 10000 & -4.0 \\
Winter & 98 & 730 & - & -11.8 \\
Spring & 65 & 400 & - & -5.0 \\
Summer & 87 & 480 & - & +6.8 \\
Autumn & 120 & 820 & - & -1.8 \\
\hline
\end{tabular}

The program stars were chosen from the input catalogue based on the CSI (Ochsenbein \& Bishoff 1975). All stars brighter than $7.2 \mathrm{mag}$ in the $V$ passband (or in the visual passband) and with declinations within $+90^{\circ}$ and $-14^{\circ}$ (the whole $-14^{\circ} \mathrm{BD}$ zone) were included into our observational program. Thus, the program covers about $63 \%$ of the sky. Some fainter stars from the HD catalogue, which were near to the main program stars, have been measured too. Additionally, we included some stars of late spectral types brighter than the 9 th magnitude. A few stars brighter than 6.5 mag have not been measured due to mistakes in the input catalogue.

Our intention was to obtain at least four measurements for each program star: two measurements with an interval $15-20$ min during one night and two other measurements during another night in other season (see Table 3, Section 7). Such a method has been accepted to control both the stability of the atmospheric extinction within a timescale of about $10 \mathrm{~min}$ and the stability of the photometric system over the whole period of observations. Also, such distribution of observations gave us a possibility to identify new variable stars and to reveal mistakes in measurements.

Two or three standard stars were usually observed each $\sim 15 \mathrm{~min}$ with the program stars. Pairs of standards with equal altitude were observed more or less periodically. To obtain the parameters of atmospheric extinction, a pair of standard stars, with a difference in air masses $\sim 0.6$, were observed two or three times per night. 
A 30 arcsec diaphragm was used most frequently. In the case of close multiple stars, we tried to measure both the total brightness and the individual components, if it was possible.

\section{THE PHOTOMETRIC EQUIPMENT}

For the photometry we used a four-channel photometer providing simultaneous measurements in $W, B, V$ and $R$ photometric bands, connected with a computer for measurement, recording and preliminary analysis of the data. The main feature of the photometer (Kornilov \& Krylov 1990) is a division of the light beam among the channels by means of semi-transparent aluminum films. Despite the losses of light, the efficiency of this photometer for measurements of stars brighter than the 9th magnitude (with a $0.5 \mathrm{~m}$ telescope) is higher than that with successive changes of filters because, in our case, the accuracy and integration time for the bright stars is defined mainly by atmospheric scintillations and practically do not depend on the brightness of a star. The use of a grey (quasi-neutral) beamsplitter, reducing the light considerably, made it possible to decrease the effect of non-linearity. In our case, the accuracy of the correction for non-linearity was better than $0.005 \mathrm{mag}$ for all stars.

The equipment for measurement, recording and preliminary analysis of the data was based on the mini-computer LSI-11 (Kornilov et al. 1990). The hardware and software used helped to increase the efficiency of photoelectric observations and to decrease the probability of wrong measurements. The main source of mistakes was in the hand-pointing of the telescope.

\section{THE PHOTOMETRIC SYSTEM}

The response functions of the WBVR system shown in Fig. 1 have been set up by means of glass filters described by Meištas et al. (1975) and kindly presented by V. Straižys. The passbands of the $U B V$ system are plotted too. The $V$ passband of Johnson's system practically coincides with the $V$ passband of our system. The transmittance curves of our filters were measured with a laboratory spectrophotometer and monochromator calibrated with an optical radiometer. The curves were reduced to a temperature of $-4^{\circ} \mathrm{C}$. 


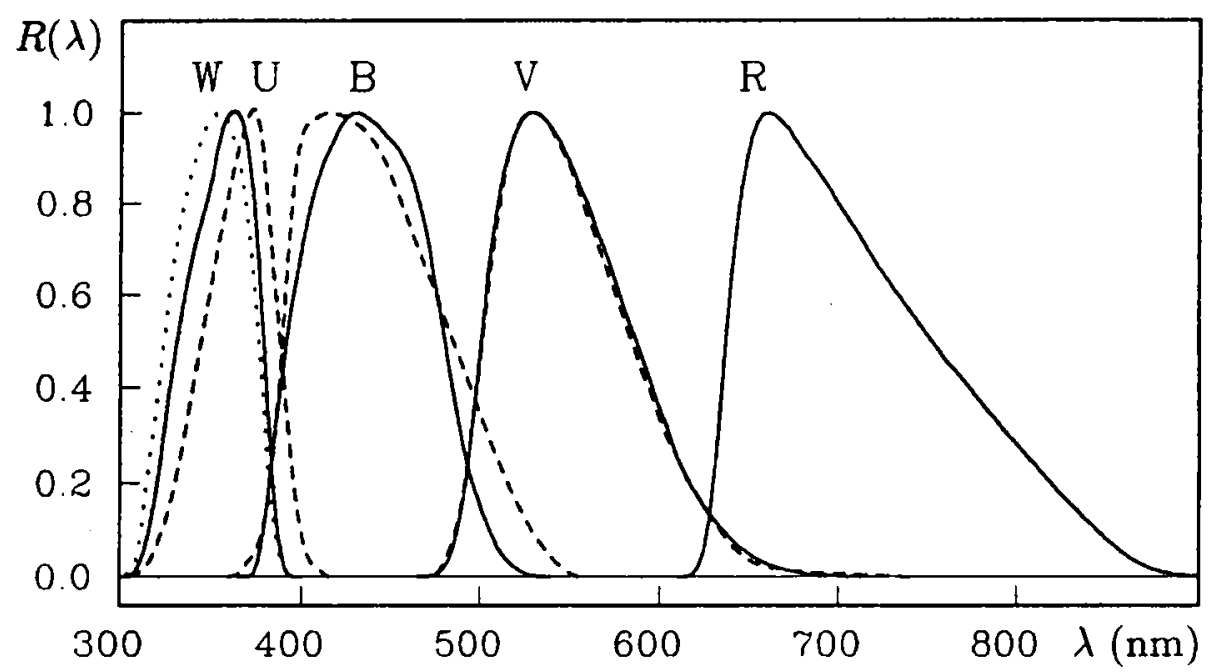

Fig. 1. Normalized response functions of the $W B V R$ (solid lines) and the $U B V$ (dashed lines) systems. The dotted line shows the response of the $W$ passband from Straižys (1977).

A network of standard stars was set up simultaneously with the observations of program stars. To define the magnitudes of 228 standard stars, more than 13000 measurements have been used.

For the definition of the zero-point of the $V$ magnitude, the star HD 5015 (F8 V) was used: for it $V=4.797 \mathrm{mag}$ is taken (Khaliullin et al. 1985). For the definition of the zero-point of color indices, 14 A0 V stars were used: their average color indices are taken to be zero. With this normalization, color indices of HD 5015 have the following values: $W-B=-0.082, B-V=+0.553$ and $V-R=+0.456$. The average color indices of six A0 stars, used for the normalization of color indices by H.L. Johnson in the $U B V$ system, are: $W-B=$ $+0.028, B-V=+0.000, V-R=-0.007$.

The magnitudes of the standards and estimates of their errors were derived simultaneously. About $50 \%$ of 192 standard stars have rms errors of $V$ magnitudes smaller than $0.0016 \mathrm{mag}, 90 \%$ of them - less than 0.0023 mag.

The basic ideas of the method used for the reduction of observations to outside the atmosphere were presented by Moshkalev \& Khaliullin (1985). Extinction of light in each passband was calculated by the numerical convolution of typical energy distributions in stellar spectra, the model curve of atmospheric extinction and the response functions of the passbands. The difference between the 

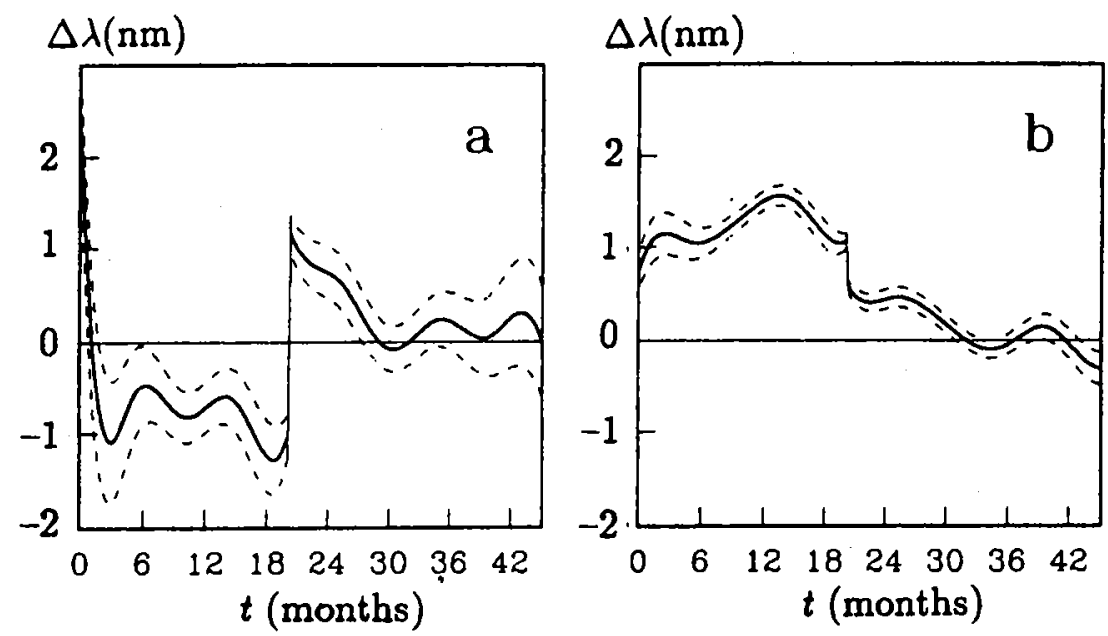

Fig. 2. The shift $\Delta \lambda$ of central wavelengths $\lambda_{0}$ for the $W$ passband (a) and for the $B$ passband (b) during the period of observations (solid line). The dashed lines indicate the rms errors of these dependences. Zero of the time axis corresponds to January 1, 1985

real extinction coefficient and the theoretical one was modeled by a two-parameter function of the wavelength. These parameters were found by minimizing the deviations of measurements of standard stars. Special attention was paid to the choice of the suitable energy distribution adequate to the observed photometric data.

\section{ANALYSIS OF OBSERVATIONAL DATA}

Some changes of the photometric system have been suspected during the preliminary data processing. These changes have affected especially the $W$ and $B$ magnitudes of the red stars. The most probable explanation of this effect is a temperature shift of the response functions. It was possible to evaluate this effect from the same observational material, since most stars have been measured in different seasons, with different outside temperatures.

However, an additional effect was found: the shift of passband with time. The detected dependence of shifts in the $W$ and $B$ passbands with time is shown in Figs. 2a and 2b. We did not find any significant changes in the $V$ and $R$ passbands. A shift of the response function by $1 \mathrm{~nm}$ causes changes of the measured magnitudes for an M0 III star up to $0.013 \mathrm{mag}$ in $W$ and $0.017 \mathrm{mag}$ in $B$. Apparently, 
these shifts can be explained by changes of spectral properties of the semitransparent aluminum layers and of the optical glue in the photometer beamsplitter. The jump in those shifts in September 1986 can be explained by the readjustment of the photometer optics and the replacement of glue in the beamsplitter.

The evaluated temperature coefficients are: $0.005,0.020,0.036$ and $0.056 \mathrm{~nm} /{ }^{\circ} \mathrm{C}$ for $W, B, V$ and $R$ passbands. The maximum change of magnitude in the $B$ passband for MO III stars is about $0.003 \mathrm{mag}$ for each $10^{\circ} \mathrm{C}$.

The data have been reduced to the fixed period (September $1987)$ and to the average temperature of observational nights $\left(-4^{\circ} \mathrm{C}\right)$. The influence of the temperature effects for the standard stars (spectral classes from A0 to G5) was small and was not taken into account. The azimuth effect did not exceed $0.003 \mathrm{mag}$ for more than $99 \%$ of standard and program stars.

The rms errors of the magnitudes were calculated for all stars except for multiple and variable stars. The errors and their correlation coefficients for different groups of stars are shown in Tables $2 \mathrm{a}$ and $2 \mathrm{~b}$. A significant correlation between the errors of $W, B$, $V$ and $R$ magnitudes is due to the simultaneous measurement in the four spectral passbands under quasi-neutral variations in atmospheric transparency. This correlation decreases the errors in the color indices.

The accuracy of photometry of binary stars with close components (the angular distance less than 5-10 arcsec; only total magnitude can be measured) or of binary stars with detached components (the angular distance more than $30-40$ arcsec; the individual components were observed) is the same as that for single stars. The errors can be larger in the intermediate cases.

\section{SOME CHARACTERISTICS OF THE CATALOG}

The accuracy class is introduced to define the quality of measurements for each star. This is only a ranking estimate, not the rms error, which is difficult to estimate since there are strong correlations of different colors and too small number of measurements for each star. Number distribution of the accuracy classes for different groups of stars is shown in Fig. 3. These distributions may be used as quantitative estimations of the quality of our observational data. The statistical characteristics of the distributions correspond to the 


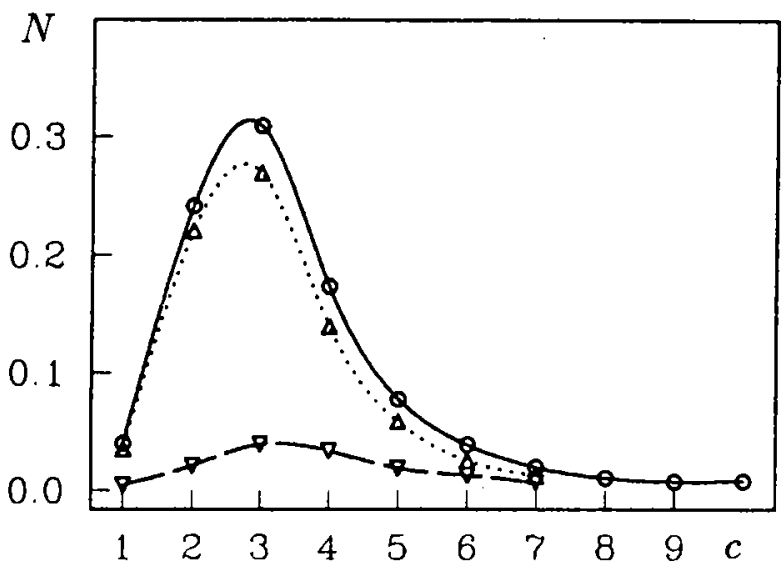

Fig. 3. The distribution of accuracy classes $c$ : circles represent all stars, triangles up are for stars brighter than $7.2 \mathrm{mag}$; triangles down are for stars fainter than $7.2 \mathrm{mag}$.

accuracy estimates of individual measurements (Table 2), taking into account that mean number of measurements for a star is 4 .

As it was mentioned above, our intention was to obtain four independent measurements for each program star. We did not succeed to do this for some stars. On the other hand, a considerable part of the stars has been measured six and more times (see Table 3 ).

The investigation of the completeness of our catalogue shows that it contains more than $95 \%$ of stars brighter than $V=7.2 \mathrm{mag}$ (see Kornilov \& Sementsov 1992). Differential star counts of the WBVR catalogue in Fig. 4 are compared with the Catalogue of Bright Stars and its Supplement (Hoffleit 1982, Hoffleit et al. 1984), the HD catalogue and the classical data by F. Seares (from Allen 1977).

\section{COMPARISON WITH THE UBV MAGNITUDES AND COL- ORS}

For a comparison of our results with magnitudes and color indices in the $U B V$ system obtained by other authors, the catalogue compiled by Mermilliod (1991) was used. The total number of common stars is 6511 , but 475 of them with the differences $V^{*}-V$ exceeding $0.1 \mathrm{mag}$ have been excluded in obtaining transformation equations (the asterisk denotes magnitudes from the Mermilliod catalogue). The correlation of magnitudes is found in the form 
Table 2a. The rms errors $\sigma$ and correlation coefficients $\rho$ in $W, B, V$ and $R$ passbands for different groups of stars.

\begin{tabular}{cccccccc}
\hline Group & $\begin{array}{l}\sigma_{W} \\
\mathrm{mag}\end{array}$ & $\begin{array}{l}\sigma_{B} \\
\mathrm{mag}\end{array}$ & $\begin{array}{l}\sigma_{V} \\
\mathrm{mag}\end{array}$ & $\begin{array}{l}\sigma_{R} \\
\mathrm{mag}\end{array}$ & $\rho_{W B}$ & $\rho_{B V}$ & $\rho_{V R}$ \\
\hline Brighter $5 \mathrm{mag}$ & $\mathbf{0 . 0 0 8}$ & $\mathbf{0 . 0 0 6}$ & $\mathbf{0 . 0 0 6}$ & $\mathbf{0 . 0 0 6}$ & 0.33 & 0.57 & 0.52 \\
$5-6.5 \mathrm{mag}$ & 0.009 & 0.006 & 0.006 & 0.006 & 0.31 & 0.45 & 0.40 \\
Fainter 6.5 mag & 0.010 & $\mathbf{0 . 0 0 7}$ & $\mathbf{0 . 0 0 7}$ & $\mathbf{0 . 0 0 8}$ & 0.29 & 0.38 & 0.29 \\
All stars & 0.010 & $\mathbf{0 . 0 0 7}$ & $\mathbf{0 . 0 0 6}$ & $\mathbf{0 . 0 0 7}$ & 0.30 & 0.41 & 0.34 \\
\hline
\end{tabular}

Table 2b. The rms errors $\sigma$ and correlation coefficients $\rho$ in $W, B, V$ and $R$ as a function of $B-R$ color indices.

\begin{tabular}{|c|c|c|c|c|c|c|c|}
\hline$B \sim R$ & $\begin{array}{c}\sigma_{W} \\
\operatorname{mag}\end{array}$ & $\begin{array}{c}\sigma_{B} \\
\operatorname{mag}\end{array}$ & $\begin{array}{l}\sigma_{V} \\
\operatorname{mag}\end{array}$ & $\begin{array}{l}\sigma_{R} \\
\mathrm{mag}\end{array}$ & $\rho_{W B}$ & $\rho_{B V}$ & $\rho_{V R}$ \\
\hline-0.25 & 0.008 & 0.006 & 0.006 & 0.008 & 0.32 & 0.47 & 0.32 \\
\hline 0.25 & 0.008 & 0.006 & 0.007 & 0.008 & 0.39 & 0.44 & 0.29 \\
\hline 0.75 & 0.009 & 0.006 & 0.006 & 0.008 & 0.41 & 0.45 & 0.29 \\
\hline 1.25 & 0.010 & 0.006 & 0.007 & 0.008 & 0.41 & 0.43 & 0.34 \\
\hline 1.75 & 0.011 & 0.007 & 0.006 & 0.007 & 0.21 & 0.40 & 0.35 \\
\hline 2.25 & 0.014 & 0.008 & 0.006 & 0.006 & 0.10 & 0.32 & 0.36 \\
\hline 2.75 & 0.019 & 0.008 & 0.006 & 0.006 & 0.07 & 0.34 & 0.44 \\
\hline 3.25 & 0.026 & 0.009 & 0.008 & 0.007 & 0.18 & 0.30 & 0.53 \\
\hline
\end{tabular}

Table 3. Statistics of the number of measurements.

\begin{tabular}{lrlll}
\hline Number of measurements & \multicolumn{2}{c}{ All stars } & \multicolumn{2}{l}{ Brighter than 7.2 mag } \\
\hline$<4$ measurements & 2782 & $21.0 \%$ & 1317 & $12.1 \%$ \\
4 measurements & 7832 & $58.6 \%$ & 7038 & $64.6 \%$ \\
$>4$ measurements & 2734 & $20.4 \%$ & 2547 & $23.3 \%$ \\
Standards & 228 & - & 218 & - \\
\hline All stars & 13586 & - & 11120 & - \\
\hline
\end{tabular}




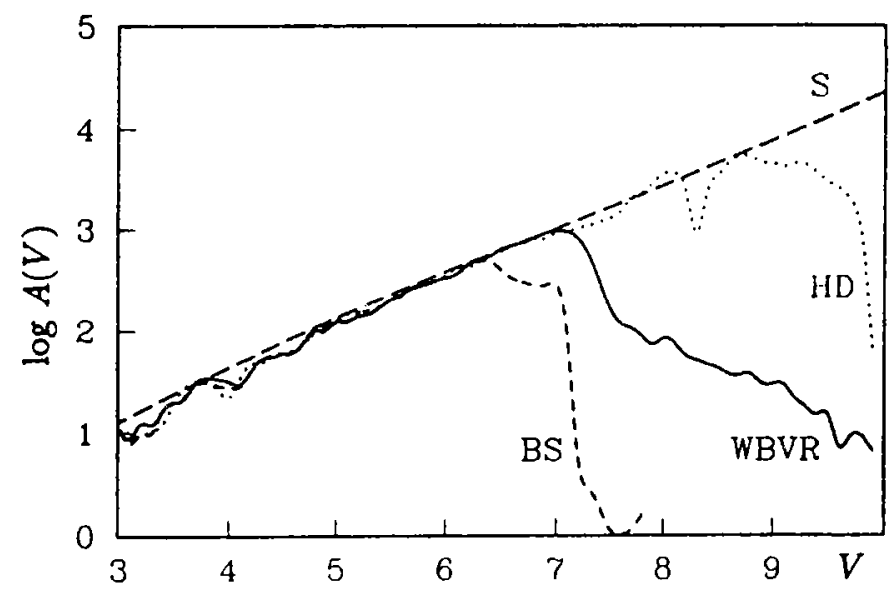

Fig. 4. Differential star counts $A_{V}$ for the $W B V R$ catalogue, the BS catalogue, the HD catalogue and the classical distribution function of F. Seares (S).

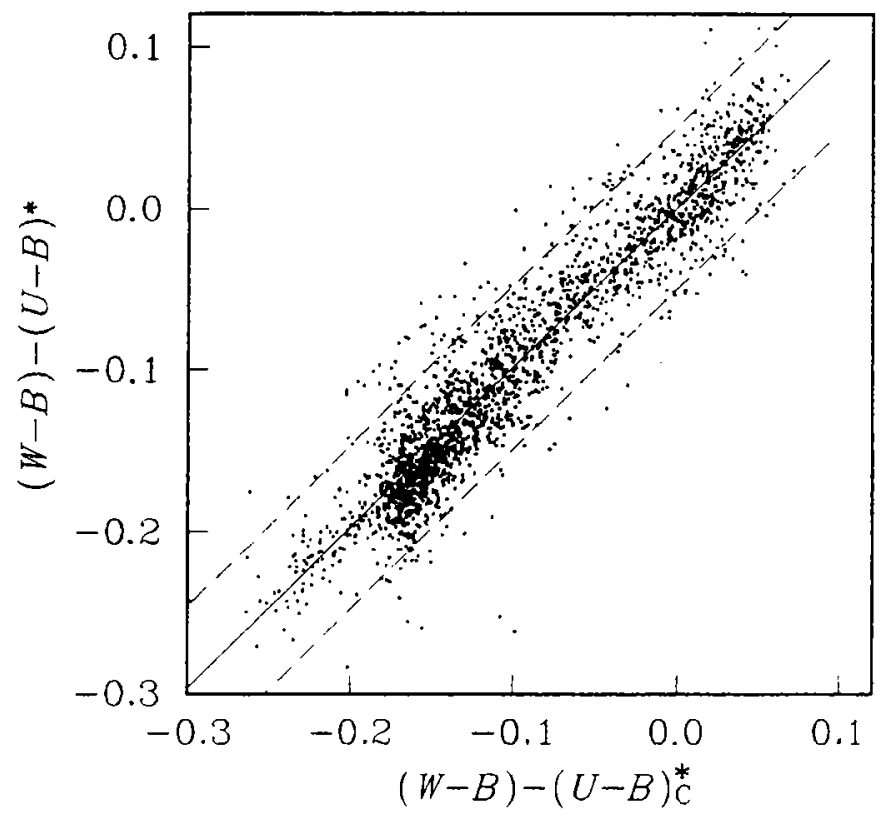

Fig. 5. Correlation between $(W-B)-(U-B)_{c}^{*}$ calculated by the transformation polinomial and the real $(W-B)-(U-B)^{*}$. The standard deviation from the regression line is $0.026 \mathrm{mag}$. Dashed lines are $95 \%$ boundaries. The correlation coefficient is 0.94 . Only a third of the stars are plotted. 


$$
V^{*}-V=-0.0019-0.061(B-V)+0.072(V-R) .
$$

The rms scatter of the residuals $\delta=V^{*}-V_{c}^{*}$ is $0.022 \mathrm{mag}$; here $V_{c}^{*}$ is the value of magnitude calculated by Equation (1). It means that: (1) the zero-points of both systems of $V$, defined by the stars with $B-V<0.7$, coincide with high accuracy, (2) the color correction does not exceed $0.01 \mathrm{mag}$ even for red stars, $(3)$ the scatter of residuals is caused by random errors of measurements, systematic errors of reduction of instrumental systems to the standard $U B V$ system and a probable micro-variability.

Transformation equations between our $B-V$ and $W-B$ color indices and the $(B-V)^{*}$ and $(U-B)^{*}$ color indices of the $U B V$ system also have been determined (Leontiev \& Zakharov 1995). The stars used in determination of the color equations were not separated into luminosity classes, and the interstellar reddening was not taken into account. The rms scatter of the residuals $(B-V)_{c}^{*}-(B-V)^{*}$ after the transformation is equal to $0.014 \mathrm{mag}$.

The rms scatter of the residuals $(U-B)_{c}^{*}-(U-B)^{*}$ is $0.026 \mathrm{mag}$. The transformation by a qubic polinomial with three color indices was used in this case. As Fig. 5 shows, no systematic residuals are present.

An independent comparison of the $W B V R$ and $U B V$ magnitudes and color indices was recently accomplished by Warren (1994).

\section{CONCLUSIONS}

The WBVR system was proposed in 1973 to replace the illdefined Johnson's system $U B V$. Unfortunately, the new system is not sufficiently known among astronomers. Therefore, more information about the system is necessary. One must realize that the replacement of the defective $U$ passband by the revised $W$ passband and the introduction of the exact transformation of the $W-B$ color index to outside the atmosphere should considerably increase its accuracy (Straižys 1973, 1977, 1983, 1992). Let us hope that homogeneity and significant volume of the $W B V R$ photometry published by the authors will be a good start for a wide use of this variant of the $U B V$ system. The complete $W B V R$ catalogue on a disk may be obtained from the authors. Soon it will be available at the Strasbourg Stellar Data Center. 


\section{REFERENCES}

Allen C.W. 1977, Astrophysical Quantities, Mir Publishers, Moscow, p. 350

Hoffleit D. 1982, The Bright Star Catalogue, 4th ed., Yale University Observatory

Hoffleit D., Saladyga M., Wlasuk P., 1984, A Supplement to the Bright Star Catalogue, Yale University Observatory

Khaliullin Kh., Mironov A.V., Moshkalev V.G., 1985, A\&SS, 111, 291

Kornilov V.G., Krylov A. V. 1990, AZh, 67, 173 = Soviet Astron., 34, 90

Kornilov V.G., Krutjakov A.N., Mironov A.V. 1990, in Photometric

Equipment of the Tien Shan Station of the Sternberg Astronomical

Institute, Moscow University Publ., p. 125

Kornilov V.G., Sementsov V.N. 1992, AZh, 69, 1315 = Soviet Astron., 36,670

Kornilov V.G., Volkov I. M., Zakharov A.I. et al. 1991, Catalogue of WBVR Magnitudes of Bright Stars of the Northern Sky, Trudy of the Sternberg Astron. Inst., Moscow University, Vol. 63

Leontiev S. E., Zakharov A.I. 1995, in preparation

Meištas E., Zdanavičius K., Straižys V., Gurklytè A. 1975, Bull. Vilnius Obs., No. 42,3

Mermilliod J.-C. 1991, Photoelectric Photometric Catalogue of Homogeneous Measurements in the UBV system, Strasbourg Stellar Data Center, S2168

Mironov A. V., Moshkalev V.G., Khaliullin Kh. 1984, Astron. Circular, Moscow, No. 1351, 1

Moshkalev V.G., Khaliullin Kh.F. 1985, AZh, 62, 393 = Soviet Astron., 29,227

Ochsenbein F., Bishoff M. 1975, Bull. Inform. CDS, Strasbourg, No. 8, 2 Straižys V. 1973, A\&A, 28, 349

Straižys V. 1977, Multicolor Stellar Photometry, Mokslas Publishers, Vilnius, p. 126

Straižys V. 1983, Bull. Inform. CDS, Strasbourg, No. 25, 41

Straižys V. 1992, Multicolor Stellar Photometry, Pachart Publishing House, Tucson, Arizona

Warren W. H. 1994, BAAS, 26, 1449 